\title{
Radiative Neutrino Model with Inert Triplet Scalar
}

\author{
Hiroshi Okada, * and Yuta Orikasa ${ }^{2,3, \dagger}$ \\ ${ }^{1}$ Physics Division, National Center for Theoretical Sciences, Hsinchu, Taiwan 300 \\ ${ }^{2}$ School of Physics, KIAS, Seoul 130-722, Korea \\ ${ }^{3}$ Department of Physics and Astronomy, \\ Seoul National University, Seoul 151-742, Korea
}

(Dated: October 2, 2018)

\begin{abstract}
We study a one-loop induced radiative neutrino model with an inert isospin triplet scalar field in the general framework of $U(1)_{Y}$, in which we discuss current neutrino oscillation data, lepton flavor violations, muon anomalous magnetic moment, and a dark matter candidate depending on the number of hypercharges. We show global analysis combining all the constraints, and discuss the model.
\end{abstract}

Keywords:

*Electronic address: macokada3hiroshi@cts.nthu.edu.tw

${ }^{\dagger}$ Electronic address: 


\section{INTRODUCTION}

Radiative seesaw models are well known as one of the economical and simultaneous explanations of tiny neutrino masses and dark matter (DM) due to having the strong correlations between them, and these issues are surely discussed beyond the standard model (SM). Even though a vast literature has recently arisen in Refs. [1 101], there are few papers including inert isospin triplet scalar fields [86, 87]. Especially, 1-hypercharge triplet scalars (even) with nonzero vacuum expectation value (VEV) provides the type-II seesaw mechanism that naturally explains the tiny neutrino masses due to the smallness of VEV, which is experimentally required. ${ }^{1}$ It also gives a lot of phenomenological aspects and/or constraints such as lepton flavor violation (LFV) processes through charged and/or neutral bosons, electroweak precision test, and the lowest bounds on their masses at large hadron collider (LHC).

In this paper, we study a one-loop induced radiative neutrino model with an inert isospin triplet boson (instead of nonzero VEV), in which we discuss neutrino oscillations, lepton flavor violations, anomalous magnetic moment, and a (singlet-like) bosonic dark matter candidate to explain the relic density and the direct detection. Then we show the several results from the global numerical analysis. Here we introduce the simplest discrete symmetry $Z_{2}$ as an additional symmetry, which differentiates between SM fields and new fields and assures the stability of DM.

This paper is organized as follows. In Sec. II, we show our model, including neutrino sector, LFVs, muon anomalous magnetic moment. In Sec. III, we analyze bosonic DM candidate to explain relic density and direct detection. In Sec. IV, we have a numerical analysis, and show some results. We conclude and discuss in Sec. V.

\section{MODEL SETUP}

In this section, we explain our model. The particle contents and their charges are shown in Tab. III. We add three (or two) iso-spin doublet vector-like exotic fermions $L^{\prime}$ with $-N / 2$ hypercharge, an isospin triplet scalar $\Delta$ with $(1+N) / 2$ hypercharge, and an isospin singlet scalar $S$ with $(-1+N) / 2$ hypercharge to the SM, where $N$ is the odd inters. Here $S$ can

\footnotetext{
${ }^{1}$ The theoretical reason has also been proposed by, i.e., the paper in Ref. 32.
} 


\begin{tabular}{|c||c|c|c||c|c|c|}
\hline \hline \multicolumn{1}{|c||}{} & \multicolumn{3}{c||}{ Lepton Fields } & \multicolumn{3}{c|}{ Scalar Fields } \\
\hline & $L_{L}$ & $e_{R}$ & $L^{\prime}$ & $\Phi$ & $\Delta$ & $S^{-1+m}$ \\
\hline$S U(2)_{L}$ & $\mathbf{2}$ & $\mathbf{1}$ & $\mathbf{2}$ & $\mathbf{2}$ & $\mathbf{3}$ & $\mathbf{1}$ \\
\hline$U(1)_{Y}$ & $-\frac{1}{2}$ & -1 & $-\frac{N}{2}$ & $\frac{1}{2}$ & $\frac{1+N}{2}$ & $\frac{-1+N}{2}$ \\
\hline
\end{tabular}

TABLE I: Contents of fermion and scalar fields and their charge assignments under $S U(2)_{L} \times U(1)_{Y}$, where $m \equiv \frac{1+N}{2}$ is the quantum number of the electric charge.

be a real field for brevity, while $\Delta$ has to be a complex field. We assume that only the SM Higgs $\Phi$ have vacuum expectation value (VEV), which is symbolized by $v / \sqrt{2}$.

The relevant Lagrangian and Higgs potential under these symmetries are given by

$$
\begin{aligned}
-\mathcal{L}_{Y} & =\left(y_{\ell}\right)_{i j} \bar{L}_{L i} \Phi e_{R j}+\left(y_{L}\right)_{i j} \bar{L}_{L_{i}} L_{R_{j}}^{\prime} S+\left(y_{\Delta}\right)_{i j} \bar{L}_{L_{i}}^{c}\left(i \tau_{2}\right) \Delta L_{L_{j}}+\left(M_{L}\right)_{i j} \bar{L}_{L i}^{\prime} L_{R j}^{\prime}+\text { h.c. }, \\
\mathcal{V} & =m_{\Phi}^{2} \Phi^{\dagger} \Phi+m_{S_{2}}^{2} S^{2}+m_{\Delta}^{2} \operatorname{Tr}\left[\Delta^{\dagger} \Delta\right] \\
& +\lambda_{0}\left(\Phi^{T}\left(i \tau_{2}\right) \Delta^{\dagger} \Phi S+\text {.h.c. }\right)+\lambda_{\Phi}\left|\Phi^{\dagger} \Phi\right|^{2}+\lambda_{S} S^{4}+\lambda_{\Delta}\left[\operatorname{Tr}\left(\Delta^{\dagger} \Delta\right)\right]^{2}+\lambda_{\Delta}^{\prime} \operatorname{Det}\left[\Delta^{\dagger} \Delta\right] \\
& +\lambda_{\Phi S}\left(\Phi^{\dagger} \Phi\right) S^{2}+\lambda_{\Phi \Delta}\left(\Phi^{\dagger} \Phi\right) \operatorname{Tr}\left[\Delta^{\dagger} \Delta\right]+\lambda_{\Phi \Delta}^{\prime} \sum_{i=1}^{3}\left(\Phi^{\dagger} \tau_{i} \Phi\right) \operatorname{Tr}\left[\Delta^{\dagger} \tau_{i} \Delta\right],
\end{aligned}
$$

where $i=1-3, j=1-3, \tau_{i}(i=1-3)$ is Pauli matrix, and the first term of $\mathcal{L}_{Y}$ can generates the SM charged-lepton masses $m_{\ell} \equiv y_{\ell} v / \sqrt{2}$ after the electroweak spontaneous breaking of $\Phi$. We work on the basis where all the coefficients are real and positive for simplicity. The scalar fields can be parameterized as

$$
\Phi=\left[\begin{array}{c}
w^{+} \\
\frac{v+\phi+i z}{\sqrt{2}}
\end{array}\right], \quad \Delta=\left[\begin{array}{cc}
\frac{\Delta^{m}}{\sqrt{2}} & \Delta^{1+m} \\
\Delta^{-1+m} & -\frac{\Delta^{m}}{\sqrt{2}}
\end{array}\right],
$$

where $m \equiv \frac{1+N}{2}$ is the quantum number of the electric charge, $v \simeq 246 \mathrm{GeV}$ is VEV of the Higgs doublet, and $w^{ \pm}$and $z$ are respectively GB which are absorbed by the longitudinal component of $W$ and $Z$ boson. Inserting the tadpole condition; $\partial \mathcal{V} /\left.\partial \phi\right|_{v}=0$, the SM Higgs mass is given by $\sqrt{2 \lambda_{\Phi}} v$. While the resulting mass eigenstate and the matrix for the inert CP even boson masses $M_{H}\left(\Delta_{R}, S\right)$ are respectively given as

$$
\begin{aligned}
& O^{T} M_{H}\left(\Delta^{-1+m}, S^{-1+m}\right) O=\left[\begin{array}{cc}
m_{H_{1}^{-1+m}}^{2} & 0 \\
0 & m_{H_{2}^{-1+m}}^{2}
\end{array}\right], \\
& {\left[\begin{array}{c}
\Delta^{-1+m} \\
S^{-1+m}
\end{array}\right]=O\left[\begin{array}{c}
H_{1}^{-1+m} \\
H_{2}^{-1+m}
\end{array}\right]=\left[\begin{array}{cc}
\cos \alpha & \sin \alpha \\
-\sin \alpha & \cos \alpha
\end{array}\right]\left[\begin{array}{l}
H_{1}^{-1+m} \\
H_{2}^{-1+m}
\end{array}\right],}
\end{aligned}
$$




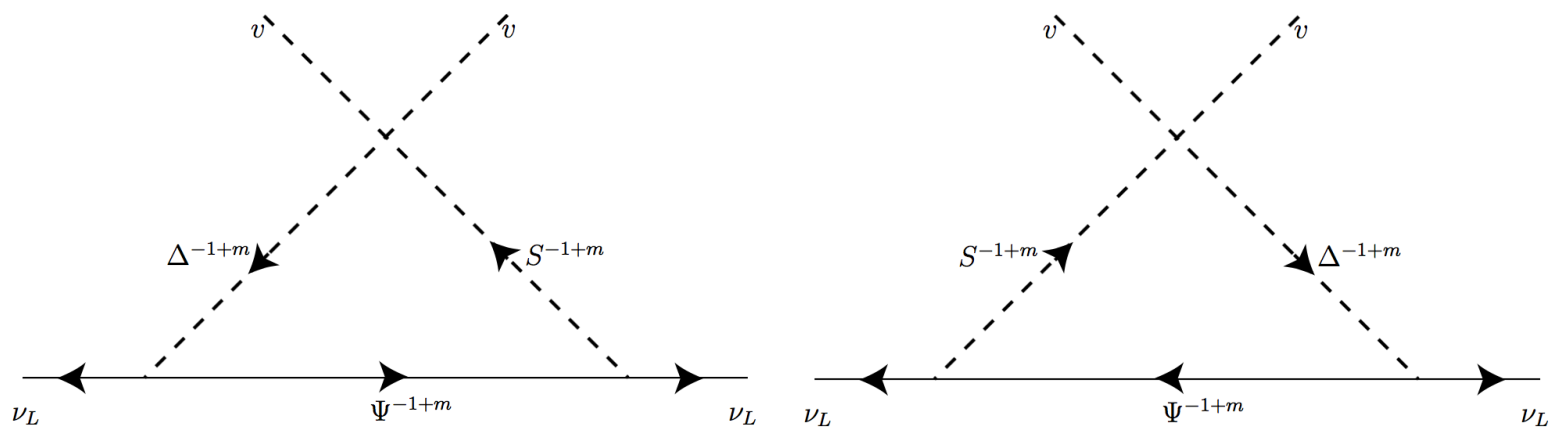

FIG. 1: Neutrino masses at the one-loop level.

with

$$
M_{H}=\left[\begin{array}{cc}
m_{\Delta}^{2}+\frac{\left(\lambda_{\Phi \Delta}+\lambda_{\Phi \Delta}^{\prime}\right) v^{2}}{2} & -\sqrt{2} \lambda_{0} v^{2} \\
-\sqrt{2} \lambda_{0} v^{2} & 2 m_{S}^{2}+\lambda_{\Phi S} v^{2}
\end{array}\right], \quad \sin 2 \alpha=\frac{2 \sqrt{2} \lambda_{0} v^{2}}{m_{H_{1}^{-1+m}}^{2}-m_{H_{2}^{-1+m}}^{2}}
$$

The other mass eigenstates is respectively given by

$$
\begin{aligned}
& m_{\Delta^{-1+m}}^{2}=m_{\Delta}^{2}+\frac{\lambda_{\Phi \Delta} v^{2}}{2}, \\
& m_{\Delta^{-1+m}}^{2}=m_{\Delta}^{2}+\frac{\left(\lambda_{\Phi \Delta}-\lambda_{\Phi \Delta}^{\prime}\right) v^{2}}{2} .
\end{aligned}
$$

\section{A. Neutrino mass matrix}

At first we redefine relevant terms in terms of the mass eigenstate as

$$
\begin{aligned}
\mathcal{L}_{Y} & \supset \frac{\left(y_{L}\right)_{i j}}{\sqrt{2}} \overline{\Psi_{R_{i}}^{-1+m}} \nu_{L_{j}}\left(-s_{\alpha} H_{1}^{-1+m}+c_{\alpha} H_{2}^{-1+m}\right) \\
& +\frac{\left(y_{\Delta}\right)_{i j}}{\sqrt{2}} \overline{\left(\Psi_{L_{i}}^{c}\right)^{1-m}} \nu_{L_{j}}\left(-c_{\alpha} H_{1}^{-1+m}+s_{\alpha} H_{2}^{-1+m}\right),
\end{aligned}
$$

where we define the isospin doublet exotic fermion as $L^{\prime} \equiv\left[\Psi^{1-m}, \Psi^{-m}\right]^{T}$.

Then the dominant contribution to the active neutrino mass matrix $m_{\nu}$ is given at oneloop level as shown in Figure 1, and its formula is given by

$$
\left(m_{\nu}\right)_{a b}=-\frac{s_{\alpha} c_{\alpha}}{2(4 \pi)^{2}} \sum\left[\left(y_{L}\right)_{a i}\left(y_{\Delta}\right)_{i b}+\left(y_{L}\right)_{b i}\left(y_{\Delta}\right)_{i a}\right] M_{L_{i}}\left[\frac{X_{1, i}}{X_{1, i}-1} \ln \left[X_{1, i}\right]-\frac{X_{2, i}}{X_{2, i}-1} \ln \left[X_{2, i}\right]\right]
$$


where we define $X_{a, i} \equiv\left(m_{H_{a}^{-1+m}} / M_{L_{i}}\right)^{2}(\mathrm{a}=1,2)$. One finds that the structure of this formula is the same as Ma model [5]. $\left(m_{\nu}\right)_{a b}$ can be generally diagonalized by the Pontecorvo-MakiNakagawa-Sakata mixing matrix $V_{\mathrm{MNS}}(\mathrm{PMNS})$ [102] as

$$
\begin{aligned}
\left(m_{\nu}\right)_{a b}= & \left(V_{\mathrm{MNS}} D_{\nu} V_{\mathrm{MNS}}^{T}\right)_{a b}, \quad D_{\nu} \equiv\left(m_{\nu_{1}}, m_{\nu_{2}}, m_{\nu_{3}}\right) \\
V_{\mathrm{MNS}} & =\left[\begin{array}{ccc}
c_{13} c_{12} & c_{13} s_{12} & s_{13} e^{-i \delta} \\
-c_{23} s_{12}-s_{23} s_{13} c_{12} e^{i \delta} & c_{23} c_{12}-s_{23} s_{13} s_{12} e^{i \delta} & s_{23} c_{13} \\
s_{23} s_{12}-c_{23} s_{13} c_{12} e^{i \delta} & -s_{23} c_{12}-c_{23} s_{13} s_{12} e^{i \delta} & c_{23} c_{13}
\end{array}\right],
\end{aligned}
$$

where we neglect the Majorana phase as well as Dirac phase $\delta$ in the numerical analysis for simplicity. The following neutrino oscillation data at 95\% confidence level [103] is given as

$$
\begin{aligned}
& 0.2911 \leq s_{12}^{2} \leq 0.3161,0.5262 \leq s_{23}^{2} \leq 0.5485,0.0223 \leq s_{13}^{2} \leq 0.0246 \\
& \left|m_{\nu_{3}}^{2}-m_{\nu_{2}}^{2}\right|=(2.44 \pm 0.06) \times 10^{-3} \mathrm{eV}^{2}, \quad m_{\nu_{2}}^{2}-m_{\nu_{1}}^{2}=(7.53 \pm 0.18) \times 10^{-5} \mathrm{eV}^{2}
\end{aligned}
$$

where we assume one of three neutrino masses is zero with normal ordering in our analysis below. The observed PMNS matrix can be realized by introducing the following parametrization. Here we can parametrize the Yukawa coupling $y_{L}$ as follows;

$$
\begin{aligned}
y_{L} & =\frac{1}{2}\left(V_{\mathrm{MNS}}^{*} D_{\nu} V_{\mathrm{MNS}}^{\dagger}+A\right) y_{\Delta}^{-1} R^{-1} \\
R_{i i} & \equiv \frac{s_{\alpha} c_{\alpha} M_{L_{i}}}{2(4 \pi)^{2}}\left[\frac{X_{1, i}}{X_{1, i}-1} \ln \left[X_{1, i}\right]-\frac{X_{2, i}}{X_{2, i}-1} \ln \left[X_{2, i}\right]\right],
\end{aligned}
$$

where $A$ is an arbitrary anti-symmetric matrix with complex values. In the numerical analysis as can be discussed later, we determine the value of $y_{L}$ to make a random plot for $y_{\Delta}, R$, and $A$.

\section{B. Lepton Flavor Violations}

$\ell_{b} \rightarrow \ell_{a} \gamma$ processes arise from the following term via one-loop diagrams

$$
\mathcal{L}_{Y} \supset-\frac{\left(y_{L}\right)_{i j}}{\sqrt{2}} \overline{\Psi_{R_{i}}^{m}} \ell_{L_{j}}\left(-s_{\alpha} H_{1}^{-1+m}+c_{\alpha} H_{2}^{-1+m}\right)+\left(y_{\Delta}\right)_{i j} \overline{\Psi_{L_{i}}^{m}} \ell_{L_{j}} \Delta^{1+m}+\frac{\left(y_{\Delta}\right)_{i j}}{\sqrt{2}} \overline{\Psi_{R_{i}}^{-1+m}} \ell_{L_{j}} \Delta^{m} .
$$

Then the branching ratio of $\operatorname{BR}\left(\ell_{b} \rightarrow \ell_{a} \gamma\right)$ is defined by

$$
\mathrm{BR}\left(\ell_{b} \rightarrow \ell_{a} \gamma\right)=\frac{48 \pi^{3} C_{b} \alpha_{\mathrm{em}}}{\mathrm{G}_{\mathrm{F}}^{2} m_{b}^{2}}\left(\left|a_{R}\right|^{2}+\left|a_{L}\right|^{2}\right)
$$




\begin{tabular}{c|c|c}
\hline Process & $(b, a)$ & Experimental bounds $(90 \%$ CL $)$ \\
\hline$\mu^{-} \rightarrow e^{-} \gamma$ & $(2,1)$ & $\operatorname{Br}(\mu \rightarrow e \gamma)<5.7 \times 10^{-13}$ \\
$\tau^{-} \rightarrow e^{-} \gamma$ & $(3,1)$ & $\operatorname{Br}(\tau \rightarrow e \gamma)<3.3 \times 10^{-8}$ \\
$\tau^{-} \rightarrow \mu^{-} \gamma$ & $(3,2)$ & $\operatorname{Br}(\tau \rightarrow \mu \gamma)<4.4 \times 10^{-8}$ \\
\hline
\end{tabular}

TABLE II: Summary of $\ell_{b} \rightarrow \ell_{a} \gamma$ process and the lower bound of experimental data [104].

where $\alpha_{\mathrm{em}} \approx 1 / 137$ is the fine-structure constant, $C_{b}=(1,1 / 5)$ for $(b=\mu, \tau), \mathrm{G}_{\mathrm{F}} \approx$ $1.17 \times 10^{-5} \mathrm{GeV}^{-2}$ is the Fermi constant, $a_{L}$ and $a_{R}$ are respectively computed as

$$
\begin{aligned}
\left(a_{R}\right)_{a b} & =-\frac{m_{\ell_{b}}}{(4 \pi)^{2}} \int d x d y d z \delta(x+y+z-1) x y \\
& \sum_{i=1}^{3}\left[\frac{m\left(y_{L}^{\dagger}\right)_{a i}\left(y_{L}\right)_{i b}}{2}\left(\frac{s_{\alpha}^{2}}{\Delta\left[m_{H_{1}^{-1+m}}, M_{L_{i}}\right]}+\frac{c_{\alpha}^{2}}{\Delta\left[m_{H_{2}^{-1+m}}, M_{L_{i}}\right]}\right)\right. \\
& +\frac{(m-1)\left(y_{L}^{\dagger}\right)_{a i}\left(y_{L}\right)_{i b}}{2}\left(\frac{s_{\alpha}^{2}}{\Delta\left[M_{L_{i}}, m_{H_{1}^{-1+m}}\right]}+\frac{c_{\alpha}^{2}}{\Delta\left[M_{L_{i}}, m_{H_{2}^{-1+m}}\right]}\right) \\
& \left.-\left(y_{\Delta}^{\dagger}\right)_{a i}\left(y_{\Delta}\right)_{i b}\left(\frac{1+m}{\Delta\left[M_{L_{i}}, m_{\Delta^{1+m}}\right]}+\frac{m}{\Delta\left[m_{\Delta^{1+m}}, M_{L_{i}}\right]}+\frac{m}{\Delta\left[M_{L_{i}}, m_{\Delta^{m}}\right]}+\frac{m-1}{\Delta\left[m_{\Delta^{m}}, M_{L_{i}}\right]}\right)\right],
\end{aligned}
$$

$$
\begin{aligned}
\left(a_{L}\right)_{a b} & =-\frac{m_{\ell_{a}}}{(4 \pi)^{2}} \int d x d y d z \delta(x+y+z-1) x z \\
& \sum_{i=1}^{3}\left[\frac{m\left(y_{L}^{\dagger}\right)_{a i}\left(y_{L}\right)_{i b}}{2}\left(\frac{s_{\alpha}^{2}}{\Delta\left[m_{H_{1}^{-1+m}}, M_{L_{i}}\right]}+\frac{c_{\alpha}^{2}}{\Delta\left[m_{H_{2}^{-1+m}}, M_{L_{i}}\right]}\right)\right. \\
& +\frac{(m-1)\left(y_{L}^{\dagger}\right)_{a i}\left(y_{L}\right)_{i b}}{2}\left(\frac{s_{\alpha}^{2}}{\Delta\left[M_{L_{i}}, m_{H_{1}^{-1+m}}\right]}+\frac{c_{\alpha}^{2}}{\Delta\left[M_{L_{i}}, m_{H_{2}^{-1+m}}\right]}\right) \\
& \left.-\left(y_{\Delta}^{\dagger}\right)_{a i}\left(y_{\Delta}\right)_{i b}\left(\frac{1+m}{\Delta\left[M_{L_{i}}, m_{\Delta^{1+m}}\right]}+\frac{m}{\Delta\left[m_{\Delta^{1+m}}, M_{L_{i}}\right]}+\frac{m}{\Delta\left[M_{L_{i}}, m_{\Delta^{m}}\right]}+\frac{m-1}{\Delta\left[m_{\Delta^{m}}, M_{L_{i}}\right]}\right)\right]
\end{aligned}
$$

$$
\Delta\left[m_{\rho}, m_{\sigma}\right] \approx x m_{\rho}^{2}+(y+z) m_{\sigma}^{2}
$$

where $M_{\Psi^{-1+m}}=M_{\Psi^{m}}=M_{L}$, and if $m_{\ell_{a}} \approx 0$, one can approximate the above formula to be

$$
\operatorname{BR}\left(\ell_{b} \rightarrow \ell_{a} \gamma\right) \approx \frac{48 \pi^{3} C_{b} \alpha_{\mathrm{em}}}{\mathrm{G}_{\mathrm{F}}^{2} m_{\ell_{b}}^{2}}\left|\left(a_{R}\right)_{a b}\right|^{2}
$$

Notice here that three body decay processes $\ell_{a} \rightarrow \ell_{b} \ell_{c} \ell_{d}$ at the one-loop box type of diagrams are negligible comparing to the $\mathrm{BR}\left(\ell_{b} \rightarrow \ell_{a} \gamma\right)$ types of LFVs [105]. 


\section{C. $\mu-e$ conversion}

The $\mu-e$ conversion rate $R$ is given by [106]

$$
\begin{aligned}
R & =\frac{\Gamma(\mu \rightarrow e)}{\Gamma_{\text {capt }}}, \\
\Gamma(\mu \rightarrow e) & =C_{\mu e}\left[\left|Z\left(b_{L}^{\gamma}-\frac{a_{R}}{m_{\ell_{b}}}\right)-b_{L}^{Z} \frac{(2 Z+N) A_{u}+(Z+2 N) A_{d}}{2\left(s_{t w} c_{t w}\right)^{2}}\right|^{2}+\left|\frac{Z a_{L}}{m_{\ell_{a}}}\right|^{2}\right]_{b=\mu, a=e},
\end{aligned}
$$

where $C_{\mu e} \equiv 4 \alpha_{\mathrm{em}}^{5} \frac{Z_{\mathrm{eff}}^{4}|F(q)|^{2} m_{\mu}^{5}}{Z}, A_{u} \equiv-\frac{1}{2}-\frac{4}{3} s_{t w}^{2}, A_{d} \equiv-\frac{1}{2}+\frac{2}{3} s_{t w}^{2}, \sin ^{2} \theta_{w} \equiv s_{t w}^{2} \approx 0.23$. The values of $\Gamma_{\text {capt }}, Z, N, Z_{\text {eff }}$, and $F(q)$ depend on the kind of nuclei. But we use Titanium, because its sensitivity will be improved by several orders of magnitude [107, 108] in near future compared to the current bound [109];

$$
R_{\mathrm{Ti}}^{\mathrm{Current} \text { bound }} \lesssim 4.3 \times 10^{-12} \rightarrow R_{\mathrm{Ti}}^{\text {Future bound }} \lesssim 10^{-18}
$$

Then these values are determined by $\Gamma_{\text {capt }}=2.59 \times 10^{6} \mathrm{sec}^{-1}, Z=22, N=26, Z_{\text {eff }}=17.6$, and $\left|F\left(-m_{\mu}^{2}\right)\right|=0.54[110]$.

$b_{L}^{V}$ has to be determined by our model, and its formula is given by

$$
\begin{aligned}
\left(b_{L}\right)_{a b}^{V} & =-\frac{1}{(4 \pi)^{2}} \int d x d y d z \delta(x+y+z-1) z(1-z) \\
& \sum_{i=1}^{3}\left[\frac{m\left(y_{L}^{\dagger}\right)_{a i}\left(y_{L}\right)_{i b}}{2}\left(\frac{s_{\alpha}^{2}}{\Delta\left[m_{H_{1}^{-1+m}}, M_{L_{i}}\right]}+\frac{c_{\alpha}^{2}}{\Delta^{V}\left[m_{H_{2}^{-1+m}}, M_{L_{i}}\right]}\right)\right. \\
& \left.-\left(y_{\Delta}^{\dagger}\right)_{a i}\left(y_{\Delta}\right)_{i b}\left(\frac{m}{\Delta^{V}\left[m_{\Delta^{1+m}}, M_{L_{i}}\right]}+\frac{m-1}{\Delta^{V}\left[m_{\Delta^{m}}, M_{L_{i}}\right]}\right)\right] \\
& \Delta^{V}\left[m_{\rho}, m_{\sigma}\right] \approx x m_{\rho}^{2}+(y+z) m_{\sigma}^{2}+z(x+z-1) m_{V}^{2}
\end{aligned}
$$

where $V \equiv(\gamma, Z)$, and $m_{\gamma}=0$, and $m_{Z} \approx 91.19 \mathrm{GeV} .{ }^{2}$

\section{Muon anomalous magnetic moment}

The muon anomalous magnetic moment (muon $g-2$ ) has been measured at Brookhaven National Laboratory. The current average of the experimental results is given by [11]

$$
a_{\mu}^{\exp }=11659208.0(6.3) \times 10^{-10} .
$$

\footnotetext{
${ }^{2}$ We would like thank referee to point out this process that gives rather strong constraint to our model.
} 


\begin{tabular}{|c||c|c|c||c|c|c|}
\hline \hline \multicolumn{1}{|c||}{} & \multicolumn{2}{c||}{ Lepton Fields } & \multicolumn{3}{c|}{ Scalar Fields } \\
\hline & $L_{L}$ & $e_{R}$ & $L^{\prime}$ & $\Phi$ & $\Delta$ & $S$ \\
\hline$S U(2)_{L}$ & $\mathbf{2}$ & $\mathbf{1}$ & $\mathbf{2}$ & $\mathbf{2}$ & $\mathbf{3}$ & $\mathbf{1}$ \\
\hline$U(1)_{Y}$ & $-1 / 2$ & -1 & $-1 / 2$ & $1 / 2$ & 1 & 0 \\
\hline$Z_{2}$ & + & + & - & + & - & - \\
\hline
\end{tabular}

TABLE III: Contents of fermion and scalar fields and their charge assignments under $S U(2)_{L} \times$ $U(1)_{Y} \times Z_{2}$.

It has been well known that there is a discrepancy between the experimental data and the prediction in the SM. The difference $\Delta a_{\mu} \equiv a_{\mu}^{\exp }-a_{\mu}^{\mathrm{SM}}$ was calculated in Ref. [112] as

$$
\Delta a_{\mu}=(29.0 \pm 9.0) \times 10^{-10}
$$

and it was also derived in Ref. [113] as

$$
\Delta a_{\mu}=(33.5 \pm 8.2) \times 10^{-10}
$$

The above results given in Eqs. (II.26) and (II.27) correspond to $3.2 \sigma$ and $4.1 \sigma$ deviations, respectively.

Our formula of muon $g-2$ can simply be given by

$$
\Delta a_{\mu} \approx-\frac{m_{\mu}}{2}\left[\left(a_{R}\right)_{22}+\left(a_{L}\right)_{22}\right]
$$

where the lower index 2 of $a_{R(L)}$ represents the muon.

\section{MODEL FEATURES}

\section{A. The case of $m=1$}

The case of $m=1$ is specific, because we accidentally have some additional terms as follows:

$$
\bar{L}_{L}^{c}\left(i \tau_{2}\right) \Delta L_{L}, \quad \bar{L}^{\prime c}\left(i \tau_{2}\right) \Delta L^{\prime}, \quad \Phi^{T}\left(i \tau_{2}\right) \Delta^{\dagger} \Phi, \quad \bar{L}_{L}^{\prime} L_{R}^{\prime} S
$$


Therefore the $m=1$ case cannot realize the general model by itself any longer as discussed in the previous section, since the neutrino masses are induced through the above first term at the tree level. Thus we impose an additional $Z_{2}$ symmetry for new fields as shown in Tab. III. As a result, all the terms are originated from the general Lagrangian, and the $Z_{2}$ symmetry plays a role in assuring the stability of DM candidates $S$ or the neutral component of $\Delta$. Here $S$ is a real field for brevity, while $\Delta$ has to be a complex field. Then all the discussions are same as the general one except the DM candidates. So we will discuss about the DM candidates below.

Relic density of Dark Matter: We have two DM candidates; the lightest of $N_{i}$ or $H_{i}$, where $N_{i}$ is the neutral component of $L^{\prime}$. However since the fermionic candidate $N$ interacts $\mathrm{Z}$ boson, it is ruled out by the experiment of direct detection searches such as LUX [114]. Hence we consider the bosonic DM candidate. Moreover, we identify DM as the isospin singlet-like boson $\mathrm{H}_{2}$ to evade the constraint of S-T-U parameter [115. Notice here that $\mathrm{H}_{2}$ is redefined by $X$, and its mass is symbolized by $M_{X}$ hereafter.

Direct detection: We have a spin independent scattering cross section with nucleon through the SM Higgs $(\phi)$ portal process and its form is simply given by

$$
\sigma_{N} \approx 0.082 \frac{\mu_{1}^{2} m_{N}^{4}}{\pi v^{2} M_{X}^{2} m_{\phi}^{4}}
$$

where $\mu_{1} \equiv-2\left(\lambda_{\Phi S}+2 \sqrt{2} \sin \alpha \lambda_{0}\right) v$, and the mass of neutron, which is symbolized by $m_{N}$, is around $0.939 \mathrm{GeV}$. LUX suggests that $\sigma_{N}$ should be less than $\mathcal{O}\left(10^{-45}\right) \mathrm{cm}^{2}$ at $\mathcal{O}(10)$ $\mathrm{GeV}$ mass range of DM.

Relic density: Our relevant processes for the thermal averaged cross section comes from annihilations of $2 X \rightarrow 2 \phi, 2 X \rightarrow \nu_{L} \bar{\nu}_{L}$, and $2 X \rightarrow \ell \bar{\ell},{ }^{3}$ and their form is given by [116, 117]

$$
\sigma v_{\mathrm{rel}} \approx \int_{0}^{\pi} \sin \theta d \theta \frac{|\bar{M}|^{2}}{16 \pi s} \sqrt{1-\frac{4 m_{f}^{2}}{s}}
$$

\footnotetext{
${ }^{3}$ Since we assume to be $\alpha<<1$, any processes including gauge bosons $Z / W$ are suppressed by $\sin ^{2} \alpha$ at least. Hence we can neglect these processes.
} 
where

$$
\begin{gathered}
|\bar{M}|^{2} \approx|\bar{M}(2 X \rightarrow 2 \phi)|^{2}+\left|\bar{M}\left(2 X \rightarrow \nu_{L} \bar{\nu}_{L}\right)\right|^{2}+|\bar{M}(2 X \rightarrow \ell \bar{\ell})|^{2}, \\
|\bar{M}(2 X \rightarrow 2 \phi)|^{2} \approx \frac{1}{2}\left|\frac{\mu_{1}}{v}-\frac{\mu_{1} \mu_{2}}{s-m_{\phi}^{2}}-\mu_{1}^{2}\left(\frac{1}{t-m_{H_{2}}^{2}}+\frac{1}{u-m_{H_{2}}^{2}}\right)\right|^{2}, \\
\left|\bar{M}\left(2 X \rightarrow \nu_{L} \bar{\nu}_{L}\right)\right|^{2} \approx 2 \sum_{a, b}^{1-3}\left|Y_{i, b}\right|^{2}\left|Y_{i, a}\right|^{2} \frac{M_{X}^{6}\left(2 M_{X}^{2}+M_{N_{b}}^{2}+M_{N_{a}}^{2}\right)}{\left(M_{X}^{2}+M_{N_{b}}^{2}\right)^{2}\left(M_{X}^{2}+M_{N_{a}}^{2}\right)^{2}} v_{\mathrm{rel}}^{2}, \\
|\bar{M}(2 X \rightarrow \ell \bar{\ell})|^{2} \approx 4 \sum_{a, b}^{1-3}\left|\left(y_{L}\right)_{i, b}\right|^{2}\left|\left(y_{L}\right)_{i, a}\right|^{2} \cos ^{4} \alpha \frac{M_{X}^{6}\left(2 M_{X}^{2}+M_{N_{b}}^{2}+M_{N_{a}}^{2}\right)}{\left(M_{X}^{2}+M_{N_{b}}^{2}\right)^{2}\left(M_{X}^{2}+M_{N_{a}}^{2}\right)^{2}} v_{\mathrm{rel}}^{2},
\end{gathered}
$$

where $Y_{i, j} \equiv\left(y_{L}\right)_{i, j} \cos \alpha+\left(y_{\Delta}\right)_{i, j} \sin \alpha$, and $\mu_{2} \equiv 6 \lambda_{\Phi} v$. Then the relic density is given by

$$
\Omega h^{2} \approx \frac{1.07 \times 10^{9}}{g_{*}^{1 / 2} M_{\mathrm{pl}}[\mathrm{GeV}] \int_{x_{f}}^{\infty}\left(\frac{a_{\mathrm{eff}}}{x^{2}}+6 \frac{b_{\mathrm{eff}}}{x^{3}}\right)},
$$

where $g_{*} \approx 100$ is the total number of effective relativistic degrees of freedom at the time of freeze-out, $M_{\mathrm{pl}}=1.22 \times 10^{19}[\mathrm{GeV}]$ is Planck mass, $x_{f} \approx 25$, and $a_{\text {eff }}$ and $a_{\text {eff }}$ are derived by expanding $\sigma v_{\text {rel }}$ in terms of $v_{\text {rel }}$ up to $v_{\text {rel }}^{2}$ as

$$
\sigma v_{\mathrm{rel}} \approx a_{\mathrm{eff}}+b_{\mathrm{eff}} v_{\mathrm{rel}}^{2} .
$$

The observed relic density reported by Planck suggest that $\Omega h^{2} \approx 0.12[118$.

\section{NUMERICAL RESULTS}

In this section, we randomly select values of the twelve parameters within the corresponding ranges

$$
\begin{aligned}
& M_{X} \in[0.1 \mathrm{TeV}, 3 \mathrm{TeV}], \quad\left(M_{E}, M_{N}, m_{H_{1}}, m_{\Delta^{ \pm}}, m_{\Delta^{ \pm \pm}}\right) \in\left[M_{X}, 5 \mathrm{TeV}\right], \\
& \delta \in[0,2 \pi], \quad \alpha \in[-0.3,0.3], \quad\left[\left(y_{\Delta}\right)_{i, j}, A\right] \in[-1,1], \text { for }(i, j)=(1,1),(1,3),(3,1), \quad(3,3), \\
& {\left[\left(y_{\Delta}\right)_{2,1}, \quad\left(y_{\Delta}\right)_{2,3}, A\right] \in[-1,-0.5],\left[\left(y_{\Delta}\right)_{1,2},\left(y_{\Delta}\right)_{3,2}\right]=A \in[0.5,1],} \\
& {\left[\left(y_{\Delta}\right)_{2,2}, \quad\left(y_{\Delta}\right)_{2,3}\right]=A \in[-0.1,0.1], \quad\left[\lambda_{0}, \lambda_{\Phi S}\right] \in[0,1],}
\end{aligned}
$$

to reproduce neutrino oscillation data, ${ }^{4} \mathrm{LFVs}$, the constraint of the direct detection searches ${ }^{5}$ and the observed relic density of DM. ${ }^{6}$ In this analysis, we are preparing 1 million sample

\footnotetext{
${ }^{4}$ In our analysis, we have used the normal ordering case. But we have checked that inverted ordering provides almost the same result.

${ }^{5}$ We conservatively take the constraint $\sigma_{N} \lesssim 10^{-45} \mathrm{~cm}^{2}$ for all the mass region of DM.

${ }^{6}$ We take allowed region to be $0.11 \leq \Omega h^{2} \leq 0.13$ instead of the exact value.
} 

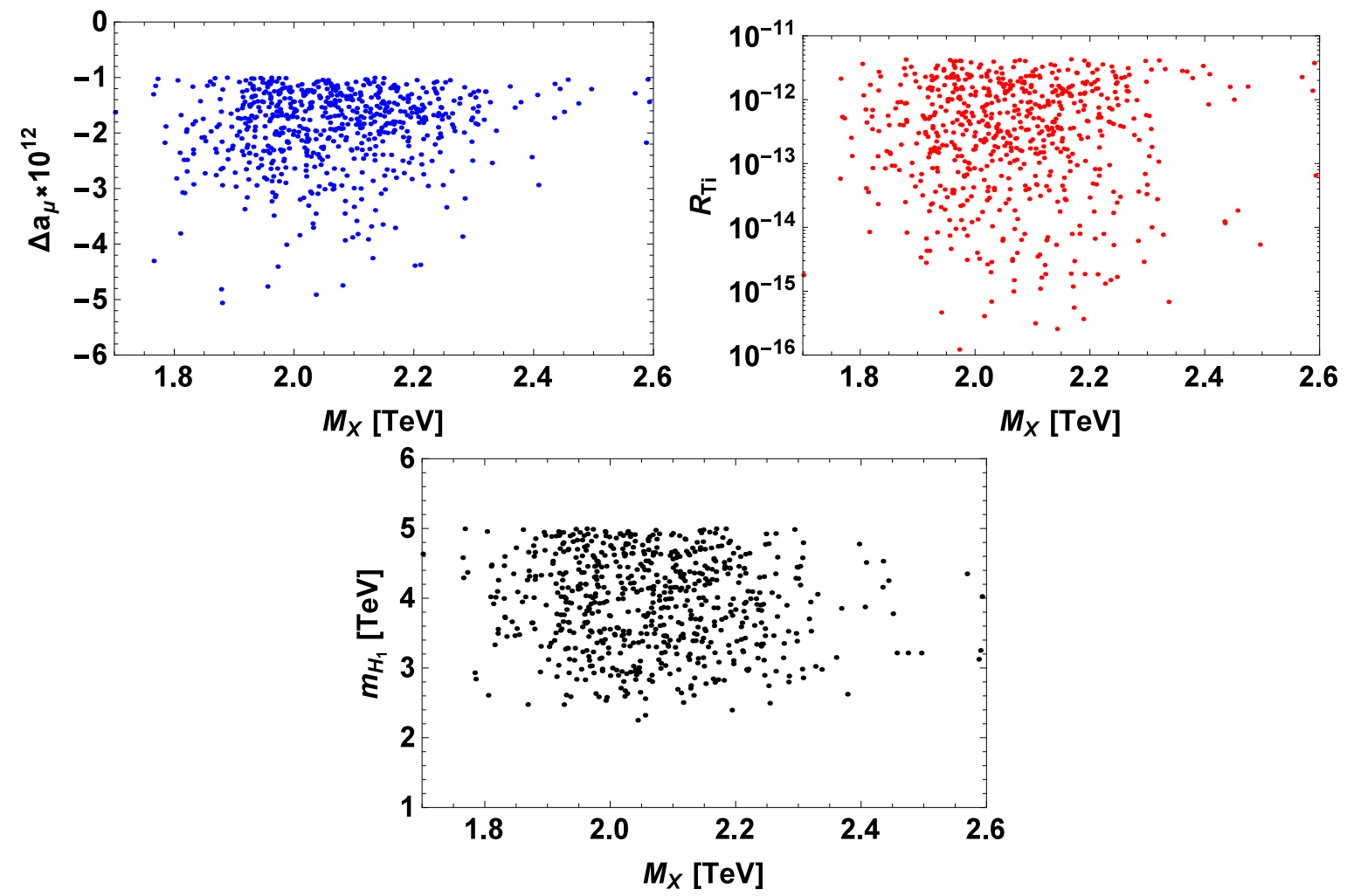

FIG. 2: Numerical results: The upper-left side figure represents the scattering plot in the DM mass and the muon anomalous magnetic moment. It suggests that the discrepancy is at most $\left|\Delta a_{\mu}\right|=\mathcal{O}\left(10^{-12}\right)$ with negative value that directly comes from the constraint of $\mu-e$ conversion rate. The allowed region of the DM mass comes from the measured relic density. The upper-right side figure represents the scattering plot in the DM mass and the $\mu-e$ conversion rate with the target $\mathrm{Ti}$ that satisfies the current upper bound. Our minimal value of $R_{\mathrm{Ti}}$ is $\mathcal{O}\left(10^{-16}\right)$, which can be tested in the future experiment that will reaches $\mathcal{O}\left(10^{-18}\right)$. The lower figure shows the scattering plot in the DM mass and the heavier inert scalar mass. It suggests that its mass should be a few times as large as the mass of DM to evade LFVs of $\ell_{b} \rightarrow \ell_{a} \gamma$.

points.

\section{A. The case $m=1$}

Here we show numerical results for $m=1$ case as shown in Figure 2. The upper-left figure represents the scattering plot in the DM mass and the muon anomalous magnetic 
moment. It suggests that the discrepancy is at most $\left|\Delta a_{\mu}\right|=\mathcal{O}\left(10^{-12}\right)$ with negative value that directly comes from the constraint of $\mu-e$ conversion rate. The upper-right side figure represents the scattering plot in the DM mass and the $\mu-e$ conversion rate with the target Ti that satisfies the current upper bound. Our minimal value of $R_{\mathrm{Ti}}$ is $\mathcal{O}\left(10^{-16}\right)$, which can be tested in the future experiment that will reaches $\mathcal{O}\left(10^{-18}\right)$. The lower side figure shows the scattering plot in the DM mass and the heavier inert scalar mass. It suggests that these masses should be a few TeV mass scale to evade LFVs and the allowed region of DM mass should be around

$$
1.7[\mathrm{TeV}] \leq M_{X} \leq 2.6[\mathrm{TeV}]
$$

which is rather narrow comparing to our scanning space. ${ }^{7}$ This result directly stems from the constraint of the relic density, and the dominant mode is $2 X \rightarrow 2 \phi$ in Eq. (III.5) due to the s-wave.

\section{B. The case of $m=2$}

We show numerical results for $m=2$ case as shown in the upper figures of 3 . The upperleft side figure represents the scattering plot in the $M_{\Psi^{ \pm}}$mass and the muon anomalous magnetic moment, and it suggests that the maximal discrepancy is $\Delta a_{\mu}=\mathcal{O}\left(1.5 \times 10^{-11}\right)$ that is below the experimental value $\mathcal{O}\left(10^{-9}\right)$ in Eq. (II.26) or Eq. (II.27). However positive value can be obtained, since the mass of $\Psi^{ \pm}$cannot be a DM candidate. The upper-right side figure represents the scattering plot in the DM mass and the $\mu-e$ conversion rate with the target $\mathrm{Ti}$ that satisfies the current upper bound. Our minimal value of $R_{\mathrm{Ti}}$ is $\mathcal{O}\left(10^{-15}\right)$, which can also be tested in the future experiment that will reaches $\mathcal{O}\left(10^{-18}\right)$. Now it is worthwhile about mentioning the way to make a decay for the exotic charged fields. $\Psi^{ \pm \pm}$can always decay into the $W^{ \pm}$boson plus $\Psi^{ \pm}$because of $S U(2)_{L}$ doublet. Then $\Psi^{2 \pm}$ can decay into the singly charged boson $S^{ \pm}$plus neutrinos through the terms $y_{L}$ or $y_{\Delta}$. Finally $S^{ \pm}$can decay into the charged leptons and the neutrinos through the terms $y_{L}$ or $y_{\Delta}$ too. Totally the series of process is as follows:

$$
\Psi^{2 \pm} \rightarrow \Psi^{ \pm}\left(+W^{ \pm}\right) \rightarrow S^{ \pm}\left(+\nu_{L}\right) \rightarrow \ell^{ \pm}+\nu_{L}
$$

\footnotetext{
7 Notice here that there exists a solution at the resonant point of the half mass of the SM Higgs boson, although we do not take this point as our range.
} 

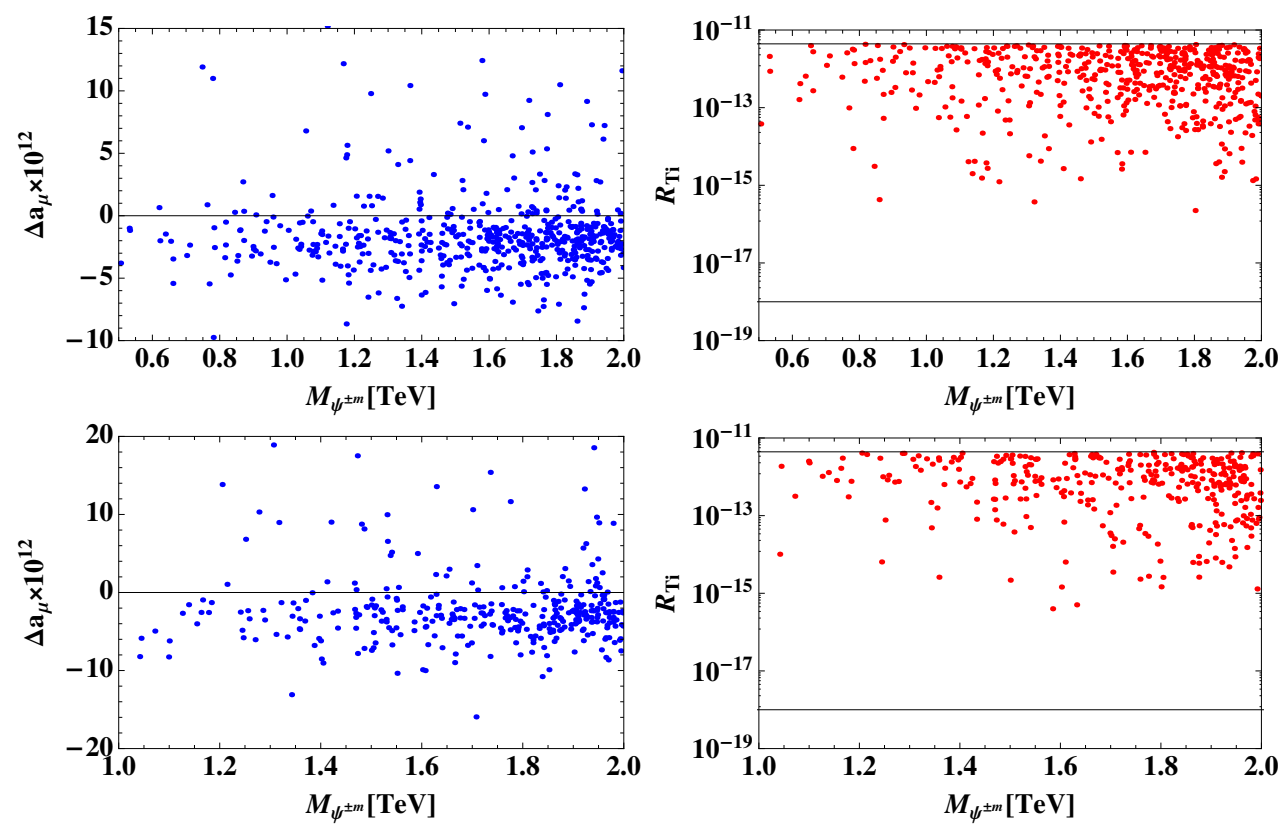

FIG. 3: The blue scattering plots represents the muon anomalous magnetic moment in terms of the exotic fermion $\left(\Psi^{ \pm}\right)$mass, where the upper figure represents $m=2$, and the lower one does $m=3$. Here the neutrino oscillation data and the LFVs are taken into consideration as constraints. These suggest that the maximal values of discrepancy are respectively $\Delta a_{\mu}=\mathcal{O}\left(1.5 \times 10^{-11}\right)$ and $\Delta a_{\mu}=\mathcal{O}\left(2.0 \times 10^{-11}\right)$ that are below the current experimental value in Eq. (II.26) or Eq. (II.27) by the order $\mathcal{O}(0.01)$. These explicitly show that the maximal value can increase, if the number of $m$ increases. However additional fields have to be introduced to make exotic fields decay into the SM fields appropriately in case where $4 \leq m$. The red scattering plots represents the $\mu-e$ conversion rate $R_{\mathrm{Ti}}$ in terms of the exotic fermion $\left(\Psi^{ \pm}\right)$mass, where the upper figure represents $m=2$, and the lower one does $m=3$. Here the neutrino oscillation data and the LFVs as well as the current upper bound of $R_{\operatorname{Ti}} \lesssim 4.3 \times 10^{-12}$ are taken into consideration as constraints. Both of the minimal values of $R_{\mathrm{Ti}}$ is $\mathcal{O}\left(10^{-16}\right)$, which will be tested in the future experiment that will reaches $\mathcal{O}\left(10^{-18}\right)$.

Thus all the exotic fields can decay into the SM fields appropriately even without the DM candidate in the case of $m=2$. 


\section{The case of $m=3$}

We show numerical results for $m=3$ case as shown in the lower figures of 3 . The lowerleft side figure represents the scattering plot in the $M_{\Psi^{ \pm}}$mass and the muon anomalous magnetic moment, and it suggests that the maximal discrepancy is $\Delta a_{\mu}=\mathcal{O}\left(2 \times 10^{-11}\right)$ that is still below the experimental value $\mathcal{O}\left(10^{-9}\right)$ in Eq. (II.26) or Eq. (II.27). However it increases comparing to the $m=2$ case by 1.5 times. The lower-right side figure represents the scattering plot in the DM mass and the $\mu-e$ conversion rate with the target Ti that satisfies the current upper bound. Our minimal value of $R_{\mathrm{Ti}}$ is $\mathcal{O}\left(10^{-15}\right)$, which can also be tested in the future experiment that will reaches $\mathcal{O}\left(10^{-18}\right)$. The decay process is following. $\Psi^{3 \pm}$ can always decay into the $W^{ \pm}$boson plus $\Psi^{2 \pm}$ because of $S U(2)_{L}$ doublet. Then $\Psi^{ \pm \pm}$ can decay into the doubly charged boson $S^{2 \pm}$ plus neutrinos through the terms $y_{L}$ or $y_{\Delta}$. Finally $S^{2 \pm}$ can decay into charged leptons with the same sign, because a new term $S^{++} \bar{e}_{R}^{c} e_{R}$ can be added into our Lagrangian. Totally the series of process is as follows:

$$
\Psi^{3 \pm} \rightarrow \Psi^{2 \pm}\left(+W^{ \pm}\right) \rightarrow S^{2 \pm}\left(+\nu_{L}\right) \rightarrow 2 \ell^{ \pm}
$$

Thus all the exotic fields can decay into the SM fields appropriately without introducing any kind of additional fields in the case of $m=3$.

\section{The case of $4 \leq m$}

In the case of $4 \leq m$, the value of muon $g-2$ can be obtained sizably, but we have to introduce new additional fields in order to make the exotic fields decay into the DM fields. For example, another singly boson $\left(h^{ \pm}\right)$and doubly boson $\left(k^{2 \pm}\right)$ have to be introduced in the case of $m=4$. Then the additional terms are written as $k^{++} \bar{e}_{R}^{c} e_{R}$ and $\bar{L}_{L}^{c} L_{L} h^{+}$. Thus the following decay process can occur:

$$
S^{3 \pm} \rightarrow k^{2 \pm}+h^{ \pm} \rightarrow 3 \ell^{ \pm}+\nu_{L}
$$

The case of $m=5$, another doubly charged boson $k^{2 \pm}$ has to be introduced. Then the following decay process can occur:

$$
S^{4 \pm} \rightarrow 2 k^{2 \pm} \rightarrow 4 \ell^{ \pm}
$$

due to the new term of $k^{++} \bar{e}_{R}^{c} e_{R}$. 


\section{CONCLUSIONS AND DISCUSSIONS}

We have studied a one-loop induced radiative neutrino model with an inert isospin triplet boson in the general framework of $U(1)_{Y}$, in which we have discussed neutrino oscillations, lepton flavor violations, anomalous magnetic moment, and a (singlet-like) bosonic dark matter candidate to explain the relic density and the direct detection (in case of $m=1$ ). Then we have found the several results from the global numerical analysis as follows. Firstly our maximal discrepancy for the muon anomalous magnetic moment are respectively given by $\left|\Delta a_{\mu}\right|=\mathcal{O}\left(10^{-12}\right)$ with negative value for $m=1, \Delta a_{\mu}=\mathcal{O}\left(1.5 \times 10^{-11}\right)$ for $m=2$, $\Delta a_{\mu}=\mathcal{O}\left(2 \times 10^{-11}\right)$ for $m=3$, where the negative sign for $m=1$ directly comes from the constraint of the $\mu-e$ conversion. Secondly the allowed region of DM mass $(m=1)$

should be around $1.7[\mathrm{TeV}] \leq M_{X} \leq 2.6[\mathrm{TeV}]$ except the resonant point, which is rather narrow comparing to our scanning space, whose result directly stems from the constraint of the relic density. Thirdly, the minimal values of $R_{\mathrm{Ti}}$ are $\mathcal{O}\left(10^{-16}\right)$ for $m=1$ and $\mathcal{O}\left(10^{-15}\right)$ for $m=(2-3)$, which will be tested in the future experiment that will reaches $\mathcal{O}\left(10^{-18}\right)$. When $4 \leq m$, we can obtain the sizable muon $g-2$, but we have found that some additional fields has to be introduced in order to make them decay into the SM fields.

The signal of our isospin triplet boson that might be measured at LHC potentially possesses the discrimination from the other radiative seesaw models, since few models introduce the triplet boson. The boson decays into the same sign dilepton + missing $(=\mathrm{DM})$ through the exotic leptons, and their corresponding Yukawa couplings $y_{\Delta}$ can always be larger than the typical values of Yukawa couplings in the type-II seesaw model because there are not any LFV processes at the tree level. It can occur when the DM mass is the resonance point at around the half mass of the SM Higgs boson, which has been discussed in ref. [45].

\section{Acknowledgments}

H. O. is sincerely grateful for all the KIAS members, Korean cordial persons, foods, culture, weather, and all the other things. This work is supported in part by NRF Research 
No. 2009-0083526 (Y. O.) of the Republic of Korea.

[1] A. Zee, Phys. Lett. B 93, 389 (1980) [Erratum-ibid. B 95, 461 (1980)].

[2] T. P. Cheng and L. F. Li, Phys. Rev. D 22, 2860 (1980).

[3] A. Zee, Nucl. Phys. B 264, 99 (1986); K. S. Babu, Phys. Lett. B 203, 132 (1988).

[4] L. M. Krauss, S. Nasri and M. Trodden, Phys. Rev. D 67, 085002 (2003) arXiv:hepph/0210389.

[5] E. Ma, Phys. Rev. D 73, 077301 (2006) hep-ph/0601225].

[6] M. Aoki, S. Kanemura and O. Seto, Phys. Rev. Lett. 102, 051805 (2009) arXiv:0807.0361.

[7] M. Gustafsson, J. M. No and M. A. Rivera, Phys. Rev. Lett. 110, 211802 (2013) arXiv:1212.4806 [hep-ph].

[8] T. Hambye, K. Kannike, E. Ma and M. Raidal, Phys. Rev. D 75, 095003 (2007) hepph/0609228.

[9] P. -H. Gu and U. Sarkar, Phys. Rev. D 77, 105031 (2008) arXiv:0712.2933 [hep-ph]].

[10] N. Sahu and U. Sarkar, Phys. Rev. D 78, 115013 (2008) arXiv:0804.2072 [hep-ph]].

[11] P. -H. Gu and U. Sarkar, Phys. Rev. D 78, 073012 (2008) arXiv:0807.0270 [hep-ph]].

[12] K. S. Babu and C. Macesanu, Phys. Rev. D 67, 073010 (2003) hep-ph/0212058.

[13] D. Aristizabal Sierra and D. Restrepo, JHEP 0608, 036 (2006) hep-ph/0604012.

[14] D. Aristizabal Sierra and M. Hirsch, JHEP 0612, 052 (2006) hep-ph/0609307.

[15] M. Nebot, J. F. Oliver, D. Palao and A. Santamaria, Phys. Rev. D 77, 093013 (2008) arXiv:0711.0483 [hep-ph]].

[16] R. Bouchand and A. Merle, JHEP 1207, 084 (2012) arXiv:1205.0008 [hep-ph]].

[17] Y. Kajiyama, H. Okada and K. Yagyu, Nucl. Phys. B 887, 358 (2014) arXiv:1309.6234 [hep-ph]].

[18] K. L. McDonald, JHEP 1311, 131 (2013) arXiv:1310.0609 [hep-ph]].

[19] E. Ma, Phys. Lett. B 732, 167 (2014) arXiv:1401.3284 [hep-ph]].

[20] D. Schmidt, T. Schwetz and H. Zhang, Nucl. Phys. B 885, 524 (2014) arXiv:1402.2251 [hep-ph]].

[21] J. Herrero-Garcia, M. Nebot, N. Rius and A. Santamaria, Nucl. Phys. B 885, 542 (2014) arXiv:1402.4491 [hep-ph]]. 
[22] A. Ahriche, S. Nasri and R. Soualah, Phys. Rev. D 89, 095010 (2014) arXiv:1403.5694 [hep-ph]].

[23] H. N. Long and V. V. Vien, Int. J. Mod. Phys. A 29, no. 13, 1450072 (2014) arXiv:1405.1622 [hep-ph]].

[24] V. Van Vien, H. N. Long and P. N. Thu, arXiv:1407.8286 [hep-ph].

[25] M. Aoki, S. Kanemura, T. Shindou and K. Yagyu, JHEP 1007, 084 (2010) [Erratum-ibid. 1011, 049 (2010)] arXiv:1005.5159 [hep-ph]].

[26] S. Kanemura, O. Seto and T. Shimomura, Phys. Rev. D 84, 016004 (2011) arXiv:1101.5713 [hep-ph]].

[27] M. Lindner, D. Schmidt and T. Schwetz, Phys. Lett. B 705, 324 (2011) arXiv:1105.4626 [hep-ph]].

[28] S. Kanemura, T. Nabeshima and H. Sugiyama, Phys. Lett. B 703, 66 (2011) arXiv:1106.2480 [hep-ph]].

[29] M. Aoki, J. Kubo, T. Okawa and H. Takano, Phys. Lett. B 707, 107 (2012) arXiv:1110.5403 [hep-ph]].

[30] S. Kanemura, T. Nabeshima and H. Sugiyama, Phys. Rev. D 85, 033004 (2012) arXiv:1111.0599[hep-ph]].

[31] D. Schmidt, T. Schwetz and T. Toma, Phys. Rev. D 85, 073009 (2012) arXiv:1201.0906 [hep-ph]].

[32] S. Kanemura and H. Sugiyama, Phys. Rev. D 86, 073006 (2012) [arXiv:1202.5231 [hep-ph]].

[33] Y. Farzan and E. Ma, Phys. Rev. D 86, 033007 (2012) arXiv:1204.4890 [hep-ph]].

[34] K. Kumericki, I. Picek and B. Radovcic, JHEP 1207, 039 (2012) arXiv:1204.6597 [hep-ph]].

[35] K. Kumericki, I. Picek and B. Radovcic, Phys. Rev. D 86, 013006 (2012) arXiv:1204.6599 [hep-ph]].

[36] E. Ma, Phys. Lett. B 717, 235 (2012) arXiv:1206.1812 [hep-ph]].

[37] G. Gil, P. Chankowski and M. Krawczyk, Phys. Lett. B 717, 396 (2012) arXiv:1207.0084 [hep-ph]].

[38] H. Okada and T. Toma, Phys. Rev. D 86, 033011 (2012) arXiv:1207.0864 [hep-ph].

[39] D. Hehn and A. Ibarra, Phys. Lett. B 718, 988 (2013) [arXiv:1208.3162 [hep-ph]].

[40] S. Baek, P. Ko, H. Okada and E. Senaha, JHEP 1409, 153 (2014) arXiv:1209.1685 [hep-ph]].

[41] P. S. B. Dev and A. Pilaftsis, Phys. Rev. D 86, 113001 (2012) arXiv:1209.4051 [hep-ph]]. 
[42] Y. Kajiyama, H. Okada and T. Toma, Eur. Phys. J. C 73, 2381 (2013) arXiv:1210.2305 [hep-ph]].

[43] M. Kohda, H. Sugiyama and K. Tsumura, Phys. Lett. B 718, 1436 (2013) arXiv:1210.5622 [hep-ph]].

[44] M. Aoki, J. Kubo and H. Takano, Phys. Rev. D 87, no. 11, 116001 (2013) arXiv:1302.3936 [hep-ph]].

[45] Y. Kajiyama, H. Okada and K. Yagyu, Nucl. Phys. B 874, 198 (2013) arXiv:1303.3463 [hep-ph]].

[46] Y. Kajiyama, H. Okada and T. Toma, Phys. Rev. D 88, 015029 (2013) arXiv:1303.7356.

[47] S. Kanemura, T. Matsui and H. Sugiyama, Phys. Lett. B 727, 151 (2013) arXiv:1305.4521 [hep-ph]].

[48] S. S. C. Law and K. L. McDonald, JHEP 1309, 092 (2013) arXiv:1305.6467[hep-ph]].

[49] B. Dasgupta, E. Ma and K. Tsumura, Phys. Rev. D 89, 041702 (2014) arXiv:1308.4138 [hep-ph]].

[50] S. Baek, H. Okada and T. Toma, JCAP 1406, 027 (2014) arXiv:1312.3761 [hep-ph]].

[51] H. Okada, arXiv:1404.0280 [hep-ph].

[52] A. Ahriche, C. S. Chen, K. L. McDonald and S. Nasri, Phys. Rev. D 90, no. 1, 015024 (2014) arXiv:1404.2696 [hep-ph]].

[53] A. Ahriche, K. L. McDonald and S. Nasri, JHEP 1410, 167 (2014) arXiv:1404.5917 [hep$\mathrm{ph}]]$.

[54] C. -S. Chen, K. L. McDonald and S. Nasri, Phys. Lett. B 734, 388 (2014) arXiv:1404.6033 [hep-ph]].

[55] S. Kanemura, T. Matsui and H. Sugiyama, Phys. Rev. D 90, 013001 (2014) arXiv:1405.1935 [hep-ph]].

[56] H. Okada and Y. Orikasa, Phys. Rev. D 90, no. 7, 075023 (2014) arXiv:1407.2543 [hep-ph]].

[57] S. Fraser, E. Ma and O. Popov, Phys. Lett. B 737, 280 (2014) arXiv:1408.4785 [hep-ph]].

[58] H. Okada, T. Toma and K. Yagyu, Phys. Rev. D 90, no. 9, 095005 (2014) arXiv:1408.0961 [hep-ph]].

[59] H. Hatanaka, K. Nishiwaki, H. Okada and Y. Orikasa, Nucl. Phys. B 894, 268 (2015) arXiv:1412.8664 [hep-ph]].

[60] S. Baek, H. Okada and K. Yagyu, JHEP 1504, 049 (2015) arXiv:1501.01530 [hep-ph]]. 
[61] L. G. Jin, R. Tang and F. Zhang, Phys. Lett. B 741, 163 (2015) [arXiv:1501.02020 [hep-ph]].

[62] P. Culjak, K. Kumericki and I. Picek, Phys. Lett. B 744, 237 (2015) arXiv:1502.07887 [hep-ph]].

[63] H. Okada, arXiv:1503.04557 [hep-ph].

[64] C. Q. Geng and L. H. Tsai, arXiv:1503.06987 [hep-ph].

[65] H. Okada, N. Okada and Y. Orikasa, arXiv:1504.01204 [hep-ph].

[66] C. Q. Geng, D. Huang and L. H. Tsai, Phys. Lett. B 745, 56 (2015) arXiv:1504.05468 [hep-ph]].

[67] A. Ahriche, K. L. McDonald, S. Nasri and T. Toma, Phys. Lett. B 746, 430 (2015) arXiv:1504.05755 [hep-ph]].

[68] D. Restrepo, A. Rivera, M. Sánchez-Peláez, O. Zapata and W. Tangarife, arXiv:1504.07892 [hep-ph].

[69] S. Kashiwase, H. Okada, Y. Orikasa and T. Toma, arXiv:1505.04665 [hep-ph].

[70] K. Nishiwaki, H. Okada and Y. Orikasa, arXiv:1507.02412 [hep-ph].

[71] W. Wang and Z. L. Han, Phys. Rev. D 92, 095001 (2015) arXiv:1508.00706 [hep-ph]].

[72] H. Okada and K. Yagyu, arXiv:1508.01046 [hep-ph].

[73] A. Ahriche, K. L. McDonald and S. Nasri, arXiv:1508.02607 [hep-ph].

[74] Y. H. Ahn and H. Okada, Phys. Rev. D 85, 073010 (2012) arXiv:1201.4436 [hep-ph]].

[75] E. Ma, A. Natale and A. Rashed, Int. J. Mod. Phys. A 27, 1250134 (2012) arXiv:1206.1570 [hep-ph]].

[76] Y. Kajiyama, H. Okada and K. Yagyu, JHEP 10, 196 (2013) arXiv:1307.0480 [hep-ph].

[77] A. E. Carcamo Hernandez, I. d. M. Varzielas, S. G. Kovalenko, H. Päs and I. Schmidt, Phys. Rev. D 88, 076014 (2013) arXiv:1307.6499 [hep-ph]].

[78] E. Ma and A. Natale, Phys. Lett. B 723, 403 (2014) arXiv:1403.6772 [hep-ph]].

[79] M. Aoki and T. Toma, JCAP 1409, 016 (2014) arXiv:1405.5870 [hep-ph]].

[80] E. Ma, Phys. Lett. B 741, 202 (2015) arXiv:1411.6679 [hep-ph]].

[81] E. Ma, arXiv:1504.02086 [hep-ph].

[82] E. Ma, Phys. Rev. Lett. 112, 091801 (2014) arXiv:1311.3213 [hep-ph]].

[83] H. Okada and K. Yagyu, Phys. Rev. D 89, 053008 (2014) arXiv:1311.4360 [hep-ph]].

[84] S. Baek, H. Okada and T. Toma, Phys. Lett. B 732, 85 (2014) arXiv:1401.6921 [hep-ph]].

[85] H. Okada and K. Yagyu, Phys. Rev. D 90, no. 3, 035019 (2014) arXiv:1405.2368 [hep-ph]]. 
[86] V. Brdar, I. Picek and B. Radovcic, Phys. Lett. B 728, 198 (2014) arXiv:1310.3183 [hep-ph]].

[87] H. Okada and Y. Orikasa, arXiv:1509.04068 [hep-ph].

[88] H. Okada, Y. Orikasa and T. Toma, arXiv:1511.01018 [hep-ph].

[89] S. Fraser, C. Kownacki, E. Ma and O. Popov, arXiv:1511.06375 [hep-ph].

[90] S. Fraser, E. Ma and M. Zakeri, arXiv:1511.07458 [hep-ph].

[91] R. Adhikari, D. Borah and E. Ma, arXiv:1512.05491 [hep-ph].

[92] S. Kanemura and H. Sugiyama, Phys. Lett. B 753, $161 \quad$ (2016) doi:10.1016/j.physletb.2015.12.012 [arXiv:1510.08726 [hep-ph]].

[93] F. Bonnet, M. Hirsch, T. Ota and W. Winter, JHEP 1207, 153 (2012) arXiv:1204.5862 [hep-ph]].

[94] D. Aristizabal Sierra, A. Degee, L. Dorame and M. Hirsch, JHEP 1503, 040 (2015) arXiv:1411.7038 [hep-ph]].

[95] H. Davoudiasl and I. M. Lewis, Phys. Rev. D 90, no. 3, 033003 (2014) arXiv:1404.6260 [hep-ph]].

[96] M. Lindner, S. Schmidt and J. Smirnov, arXiv:1405.6204 [hep-ph].

[97] H. Okada and Y. Orikasa, arXiv:1412.3616 [hep-ph].

[98] J. March-Russell, C. McCabe and M. McCullough, JHEP 1003, 108 (2010) arXiv:0911.4489 [hep-ph]].

[99] S. F. King, A. Merle and L. Panizzi, arXiv:1406.4137 [hep-ph].

[100] Y. Mambrini, S. Profumo and F. S. Queiroz, arXiv:1508.06635 [hep-ph].

[101] S. Baek and H. Okada, arXiv:1403.1710 [hep-ph].

[102] Z. Maki, M. Nakagawa, S. Sakata, Prog. Theor. Phys. 28 (1962) 870.

[103] K.A. Olive et al. (Particle Data Group), Chin. Phys. C, 38, 090001 (2014).

[104] J. Adam et al. [MEG Collaboration], Phys. Rev. Lett. 110, 201801 (2013) arXiv:1303.0754 [hep-ex]].

[105] T. Toma and A. Vicente, JHEP 1401, 160 (2014) doi:10.1007/JHEP01(2014)160 arXiv:1312.2840, arXiv:1312.2840 [hep-ph]].

[106] J. Hisano, T. Moroi, K. Tobe and M. Yamaguchi, Phys. Rev. D 53, 2442 (1996) doi:10.1103/PhysRevD.53.2442 hep-ph/9510309].

[107] E. V. Hungerford [COMET Collaboration], AIP Conf. Proc. 1182, 694 (2009). doi:10.1063/1.3293903 
[108] Y. G. Cui et al. [COMET Collaboration], KEK-2009-10.

[109] C. Dohmen et al. [SINDRUM II Collaboration], Phys. Lett. B 317, 631 (1993). doi:10.1016/0370-2693(93)91383-X

[110] R. Alonso, M. Dhen, M. B. Gavela and T. Hambye, JHEP 1301, 118 (2013) doi:10.1007/JHEP01(2013)118 arXiv:1209.2679 [hep-ph]].

[111] G. W. Bennett et al. [Muon G-2 Collaboration], Phys. Rev. D 73, 072003 (2006) hep$\mathrm{ex} / 0602035$.

[112] F. Jegerlehner and A. Nyffeler, Phys. Rept. 477, 1 (2009) [arXiv:0902.3360 [hep-ph]].

[113] M. Benayoun, P. David, L. Delbuono and F. Jegerlehner, Eur. Phys. J. C 72, 1848 (2012) arXiv:1106.1315 [hep-ph]].

[114] D. S. Akerib et al. [LUX Collaboration], Phys. Rev. Lett. 112, 091303 (2014) arXiv:1310.8214 [astro-ph.CO]].

[115] J. Beringer, et al., Phys. Rev. D 86 (2012) 010001.

[116] K. Griest and D. Seckel, Phys. Rev. D 43, 3191 (1991).

[117] J. Edsjo and P. Gondolo, Phys. Rev. D 56, 1879 (1997) hep-ph/9704361.

[118] P. A. R. Ade et al. [Planck Collaboration], Astron. Astrophys. (2014) arXiv:1303.5076 [astroph.CO]].

[119] C. Arina, R. N. Mohapatra and N. Sahu, Phys. Lett. B 720, 130 (2013) arXiv:1211.0435 [hep-ph]]. 